\title{
Expanding the scope of alkyne-mediated bioconjugations utilizing unnatural amino acids
}

Johnathan C. Maza

Coll William \& Mary, Dept Chem, Williamsburg, VA 23187 USA

Zachary M. Nimmo

Coll William \& Mary, Dept Chem, Williamsburg, VA 23187 USA

Douglas D. Young

Coll William \& Mary, Dept Chem, Williamsburg, VA 23187 USA

Follow this and additional works at: https://scholarworks.wm.edu/aspubs

\section{Recommended Citation}

Maza, J. C., Nimmo, Z. M., \& Young, D. D. (2016). Expanding the scope of alkyne-mediated bioconjugations utilizing unnatural amino acids. Chemical Communications, 52(1), 88-91.

This Article is brought to you for free and open access by the Arts and Sciences at W\&M ScholarWorks. It has been accepted for inclusion in Arts \& Sciences Articles by an authorized administrator of W\&M ScholarWorks. For more information, please contact scholarworks@wm.edu. 


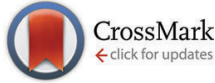

Cite this: Chem. Commun., 2016, 52,88

Received 7th October 2015,

Accepted 20th October 2015

DOI: $10.1039 / \mathrm{c} 5 \mathrm{cc} 08287 \mathrm{k}$

www.rsc.org/chemcomm

\section{Expanding the scope of alkyne-mediated bioconjugations utilizing unnatural amino acids $\dagger$}

\author{
Johnathan C. Maza, Zachary M. Nimmo and Douglas D. Young*
}

The importance of bioconjugates within the field of chemistry drives the need for novel methodologies for their preparation. Well-defined and stable bioconjugates are easily accessible via the utilization of unnatural amino acids (UAAs). As such, we have synthesized and incorporated two new UAAs into green fluorescent protein, and optimized a novel Cadiot-Chodkiewicz bioconjugation, effectively expanding the toolbox of chemical reactions that can be employed in the preparation of bioconjugates.

Bioconjugates represent a class of molecules in which a biomacromolecule is linked to another molecule, typically a probe, a surface, or a cytotoxic compound. ${ }^{1,2}$ Protein-based bioconjugates, in which a protein is the biomolecule, represent an ever-expanding field of research. Specifically, protein-based bioconjugates are becoming increasingly popular for "lab-on-a-chip" technologies, in which a diagnostic protein is immobilized on a surface, as well as towards the development of novel cancer therapeutics via the preparation of antibody-drug conjugates. ${ }^{3-5}$

The methods to generate a bioconjugate range from noncovalent interactions, typically adsorption and encapsulation, to covalent linkages. ${ }^{6}$ While a covalent attachment is more robust, and less easily disrupted in a biological setting, obtaining a degree of specificity during the synthesis has precluded its widespread application. This is a consequence of often utilizing reactive residues within the protein of interest. However, there are typically multiple nucleophilic residues that can react within the protein. ${ }^{1}$ The result is a non-specific coupling that can either disrupt normal protein function, result in improper orientation of the protein-bioconjugate, or lead to heterogeneous mixtures of linkages at multiple residues. ${ }^{6}$ These limitations can be overcome by site-specifically incorporating UAAs with chemical functionalities not found in the canonical amino acids. ${ }^{7,8}$ In particular, the suppression of the amber (TAG) stop codon via

Department of Chemistry, College of William \& Mary, Williamsburg, Virginia, 23187, USA. E-mail: dyoung01@wm.edu

$\dagger$ Electronic supplementary information (ESI) available: Experimental protocols, bioconjugation optimization and SDS-PAGE analysis. See DOI: 10.1039/c5cc08287k an exogenous amino-acyl tRNA synthetase (aaRS)/tRNA pair allows a high level of control over the position of the UAA. ${ }^{9}$ These UAA-containing proteins can then be conjugated to other molecules via a bioorthogonal reaction that occurs at physiological conditions $\left(\mathrm{pH}=7,37{ }^{\circ} \mathrm{C}\right.$ ) with no chance of crossreaction with other biomolecules. ${ }^{10,11}$

Indeed a variety of bioorthogonal reactions have already been developed. These include the copper(I)-catalyzed cycloaddition between azides and terminal alkynes, ${ }^{12-14}$ the strain-promoted cycloaddition, ${ }^{15-17}$ the oxime formation, ${ }^{18-21}$ and more recently, the formation of a conjugated diyne via the copper(I)-catalyzed Glaser-Hay coupling. ${ }^{22}$ Despite the wide array of bioorthogonal chemistries available, each possessing advantages and disadvantages, the individual requirements of the bioconjugate help dictate which reaction may be best to employ. Due to the increasing application of bioconjugates, the development of novel bioorthogonal reactions (and UAAs with which they can be employed) are at the forefront of the field.

The Cadiot-Chodkiewicz reaction affords a conjugated diyne via the reaction of a terminal alkyne and a halo-alkyne (Fig. 1). In the presence of a copper(I) salt and a monodentate nitrogenous ligand, usually triethylamine (TEA), the reaction proceeds to form a covalent linkage in the form of a conjugated diyne in a relatively chemoselective fashion. ${ }^{23,24}$ Furthermore, the overall reaction is net redox neutral, as a single copper(I) catalyst goes through a series of oxidative additions and reductive eliminations with the bromoalkyne and terminal alkyne reactant to yield the conjugated diyne. ${ }^{25}$

The Cadiot-Chodkiewicz reaction has diverse applications to many areas of chemistry. It has been utilized in polymerization reactions, such as in the formation of the backbone of a solid-state polymer crystal, or in the fabrication of polymerized monolayer assemblies. ${ }^{26,27}$ Additionally, several acetylenic natural products exhibiting valuable biological properties can be obtained via a Cadiot-Chodkiewicz reaction. ${ }^{28}$

Similar to the Cadiot-Chodkiewicz reaction, the Glaser-Hay coupling of two terminal alkynes also affords a diyne. ${ }^{29,30}$ The reaction brings together two terminal alkynes generate a diyne linkage; however, it is not redox neutral and has chemoselectivity 
A.

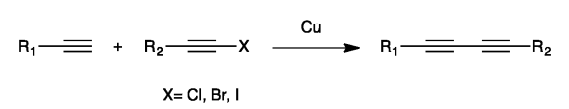

B.
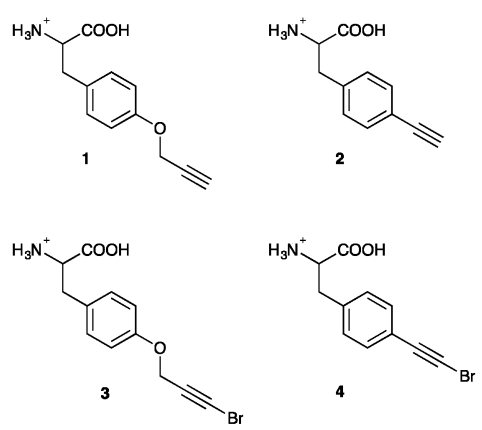

Fig. 1 Cadiot-Chodkiewicz reaction and associated UAAs. (A) Standard Cadiot-Chodkiewicz reaction employing a copper(I) salt to couple a terminal alkyne to a haloalkyne, affording an asymmetrical conjugated diyne product. (B) $p$-Propargyloxyphenylalanine (1,pPrF), p-ethynylphenylalanine (2,pEtF), p-bromopropargyloxyphenylalanine (3, pBrPrF), p-bromoethynylphenylalanine (4, pBrEtF) incorporated into GFP-151 for Glaser-Hay and CadiotChodkiewicz reactions.

issues as nothing differentiates the two terminal alkynes. ${ }^{31}$ The reaction involves the addition of a bidentate nitrogenous ligand, triethylmethylenediamine (TMEDA), ${ }^{32}$ which lowers the reaction temperatures and enhances the kinetics of the reaction. Recently, we demonstrated a bioorthogonal variant of the Glaser-Hay reaction that can be conducted in an aqueous setting under physiological conditions. ${ }^{22}$ Using a terminal alkynecontaining UAA, we were able to demonstrate that the reaction proceeds to completion within approximately $6 \mathrm{~h}$ at $4{ }^{\circ} \mathrm{C}$, with near quantitative conjugation. As previously mentioned, the Glaser-Hay coupling of terminal alkynes has a chemoselectivity issue when the terminal alkynes differ, resulting in the formation of unwanted homodimers. However, due to the steric bulk of the protein, we found that the homodimerization of the reaction was mostly inhibited, leading to primarily the desired protein heterodimer product. As such we were able to demonstrate that the Glaser-Hay reaction could be employed as a novel bioorthogonal chemistry, yielding stable conjugates with well-defined geometries. However, the reaction was limited by the oxidative damage of the protein due to the mechanistic cycling of the copper through three different redox states. As a result, reactions proceeding for longer than $6 \mathrm{~h}$, resulted in oxidative damage and protein degradation.

A key component of the Glaser-Hay mechanism is the formation of a copper(II)-hydroxyl intermediate, which has been shown to produce hydroxyl radicals that are deleterious to living systems. ${ }^{33}$ Because the copper(I) of the Cadiot-Chodkiewicz reaction is not thought to utilize copper(II) intermediates, we reasoned that the chemistry could be employed in a biological context to minimize previously observed oxidative damage. Furthermore, the reaction is highly chemoselective, as the use of a halo-alkyne minimizes the formation of homodimer side products by differentiating the two alkynes. Ultimately, this has the potential to increase the yield of the conjugated protein product.
Based on these facts and the limitations of the bioorthogonal Glaser-Hay, we sought to develop a bioorthogonal variant of the Cadiot-Chodkiewicz reaction that could be conducted in an aqueous setting and under physiological conditions.

In order to conduct and optimize a Cadiot-Chodkiewicz bioconjugation, new UAAs harboring a terminal haloalkyne needed to be synthesized and incorporated into a protein. In order to probe UAA-dependent effects on the reaction, aliphatic and aromatic brominated alkynyl UAAs were prepared from the previously reported protected $p$-propargyloxyphenylalanine $(p \operatorname{PrF}, 1)$ and the $p$-ethynylphenylalanine $(p \mathrm{EtF}, 2)$ respectively. ${ }^{34,35}$ Gratifyingly, the well-established bromination of phenylacetylene using $N$-bromosuccinamide (NBS) and silver nitrate worked well for the synthesis of both UAAs in moderate yields. ${ }^{36,37}$ Following deprotection, the final UAAs, $p$-bromo-propargyloxyphenylalanine $(p \mathrm{BrPrF}, 3)$ and $p$-bromo-ethynylphenylalanine $(p \mathrm{BrEtF}, 4)$, were recovered in overall good yields (67\% and $34 \%$ respectively) (Fig. 1).

With the $p \mathrm{BrPrF}$ and $p \mathrm{BrEtF}$ in hand, it was imperative to incorporate these UAAs into a model protein. Due to both its fluorescent properties and well-documented prior use in UAA development technologies, green fluorescent protein (GFP) was selected as a model system. Specifically, attempts were made to incorporate newly synthesized UAAs at residue 151 by suppressing the amber stop codon. Furthermore, our previous work immobilizing GFP revealed that this surface exposed site is ideal for UAA placement, as the rigidity of the residue helps orient the bioorthogonal functional handle. ${ }^{38}$ In lieu of undergoing a tedious aaRS selection process, we hoped to incorporate the brominated UAAs using the previously described promiscuous $p$ CNF aaRS. ${ }^{39,40}$ The $p$ CNF aaRS was investigated first to incorporate $\mathrm{pBrPrF}$ and $p$ BrEtF due to their structural similarity to other UAAs that the $p$ CNF aaRS incorporates.

BL21(DE3) E. coli were co-transformed with a pEVOL- $p$ CNF plasmid and a pET-GFP-TAG151 plasmid and used to initiate an expression culture at $\mathrm{OD}_{600} 0.1$ which was grown to an $\mathrm{OD}_{600}$ 0.7. The culture was subsequently centrifuged and the cell pellet was resuspended in $4 \mathrm{~mL}$ of LB broth supplemented with antibiotics, IPTG, arabinose, and the presence or absence of a UAA. ${ }^{34}$ This previously reported expression protocol allowed for the minimization of the amount of UAA employed, and was found to be very effective. After $18-20 \mathrm{~h}$ at $37{ }^{\circ} \mathrm{C}$, cells were pelleted and the expressed GFP was purified.

Gratifyingly, the promiscuous $p \mathrm{CNF}$ aaRS incorporated both brominated-UAA variants with a higher fidelity than the simple terminal alkyne analogs (Fig. 2). As is to be expected, the smaller $p$ BrEtF UAA, had a higher incorporation than the $p$ BrPrF. We hypothesize that the 1-bromo-alkyne moiety provides a degree of hydrophobic character to the UAA, making the interaction between the amino acid and the hydrophobic binding pocket of the aaRS more favourable.

With both brominated alkyne UAAs in hand, it was feasible to develop a bioorthogonal Cadiot-Chodkiewicz reaction. Initial studies employed the $p$ BrPrF-containing GFP variant, to mimic previous Glaser-Hay $p$ PrF-GFP studies and provide an effective comparison. The Cadiot-Chodkiewicz reaction was carried out in PBS $(\mathrm{pH}=7)$ using copper iodide and triethylamine (TEA) 
A

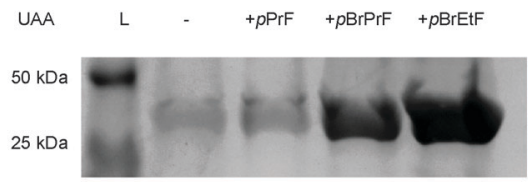

B

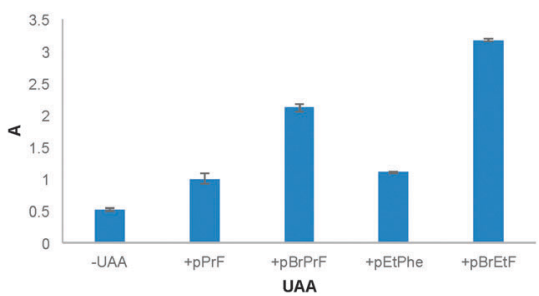

Fig. 2 Expression of UAA-containing GFP151 using the promiscuous $p C N F$ aaRS. (A) A stained SDS-PAGE gel indicated successful incorporation of $\mathbf{3}$ and $\mathbf{4}$ over background (-). A positive control, 1, was also utilized in an expression. (B) Data for overall incorporation of UAAs via the $p C N F$ aaRS as measured via absorbance at $280 \mathrm{~nm}$ on a nanodrop spectrophotometer $\left(\varepsilon / 1000=20, M_{W}=26.80 \mathrm{kDa}\right)$. Data obtained were normalized to the $p \operatorname{PrF}$ UAA (1) to demonstrate the difference in incorporation efficiency over the previously reported UAA.

(both at a final concentration of $5 \mathrm{mM}$ ) in the presence of a terminal alkyne-containing fluorophore (AlexaFluor 488 alkyne) at $4{ }^{\circ} \mathrm{C}$ for $6 \mathrm{~h}$. The reaction was successful, as fluorescence could be detected on a denatured SDS-PAGE gel only when protein and fluorophore were exposed to the CuI/TEA system (Fig. 3). Even more exciting was the minimal protein degradation relative to the previously reported Glaser-Hay reaction. Also, due to the chemoselective nature of the reaction no protein dimerization was detected, and fluorophore dimerization was minimal and easily removed.

In an attempt to further optimize the reaction, both copper concentrations and temperatures were varied. A $5 \mathrm{mM}$ working $\mathrm{Cu}(\mathrm{I})$ concentration was found to be ideal, which represents a marked improvement over the $\sim 50 \mathrm{mM}$ concentrations required for the Glaser-Hay reaction (see ESI $\dagger$ ). Previous reports have indicated that in vivo use of copper-mediated bioorthogonal chemistries required working concentrations of near $0.1 \mathrm{mM}$ of copper(I) salt to minimize cytotoxicity. ${ }^{10,41}$ Thus, the minimized copper concentrations help bring bioorthogonal conjugated diyne chemistry into the range of in vivo use. These copper concentrations also had no impact on GFP fluorescence as determined by control reactions. Additionally, the optimal temperature profile for the reaction was also investigated. After performing a time course of the reaction at both $37{ }^{\circ} \mathrm{C}$ and $4{ }^{\circ} \mathrm{C}$, we were able to determine very little difference between either temperature at early time-points. However, as the reaction was extended to $24 \mathrm{~h}$, greater protein degradation at $37{ }^{\circ} \mathrm{C}$ occurred, most likely due to an increase in the rate of disproportionation of the $\mathrm{Cu}(\mathrm{I})$ catalyst at this temperature, producing a reactive copper(II) species (Fig. 3 and ESI $\dagger$ ). However, for the $4{ }^{\circ} \mathrm{C}$ temperature profile the reaction reached approximately $86 \%$ completion in $4 \mathrm{~h}$ with minimal protein degradation, indicating that the bioorthogonal Cadiot-Chodkiewicz reaction can be performed quickly and in a relatively mild conditions. Extended times and temperatures resulted in higher yields, however were accompanied by protein degradation.
A

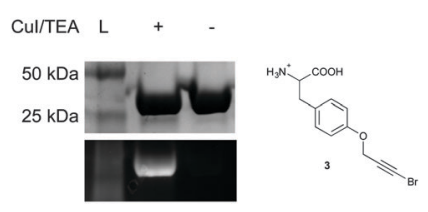

B

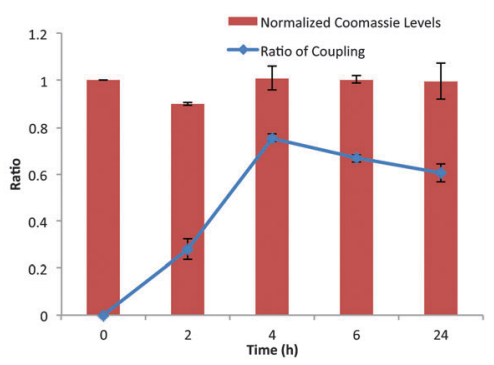

Fig. 3 Bioorthogonal Cadiot-Chodkiewicz reaction. (A) The reaction performed on GFP $\left(0.2 \mathrm{mg} \mathrm{mL}^{-1}\right)$ and AlexaFluor $488(0.2 \mathrm{mM})$ in the presence of $5 \mathrm{mM}$ of $\mathrm{Cul}$ and $5 \mathrm{mM}$ TEA at $4{ }^{\circ} \mathrm{C}$. Fluorescence is only observed in the presence of the Cul/TEA system. (B) Reaction profile at $4{ }^{\circ} \mathrm{C}$ over a $24 \mathrm{~h}$ time period. Following analysis via SDS-PAGE, fluorescent imaging, and staining with coomassie blue, protein levels were normalized to the 0 time point control. The protein levels indicate minimal to no protein degradation occurs, even at longer time points. Analysis of coupling efficiency was determined by calculating the ratio of fluorescence to coomassie staining for each time point. All reactions were performed in triplicate.

We next sought to explore the effects of an aromatic variant of the pBrPrF. As such, $p$ BrEtF-GFP151 was expressed, and subjected to coupling conditions at $4{ }^{\circ} \mathrm{C}$ in the presence of an alkyne fluorophore. Once again a successful conjugation was observed as determined by SDS-PAGE (see ESI $\dagger$ ). Only samples exposed to the CuI/TEA system exhibited fluorescence while other controls did not, indicating the fluorescence was not due to non-specific interactions. Interestingly, the use of an aromatic containing bromoalkyne appears to be less effective in the Cadiot-Chodkiewicz reaction than its aliphatic analog.

Next we became interested in exploring how the novel biological Cadiot-Chodkiewicz conjugation compared to our previously described Glaser-Hay reactions. In direct comparison, the CadiotChodkiewicz exhibited far less protein degradation as compared to the Glaser-Hay versions of either an aliphatic $(p \mathrm{PrF})$ or aromatic ( $p$ EtF) terminal alkyne containing UAAs. Furthermore, the data indicates that the biological Cadiot-Chodkiewicz reaction proceeds at a faster rate than the Glaser-Hay, with the aliphatic version ( $p$ BrPrF) of the coupling reaching completion the fastest in $4 \mathrm{~h}$ (Fig. 4). Gratifyingly, these results correlate well with the mechanistic understanding of both reactions, as the Cadiot-Chodkiewicz requires a single copper atom, while the Glaser-Hay necessitates two copper-alkyne conjugates to form the diyne product. Moreover, the $\mathrm{Cu}(\mathrm{I}) /(\mathrm{III})$ redox couple of the Cadiot-Chodkiewicz reaction most likely aids in the minimized protein oxidation relative to the GlaserHay coupling that involves a reactive $\mathrm{Cu}(\mathrm{II})$ intermediate. However, it is important to note that the Cadiot-Chodkiewicz requires brominated UAAs, requiring additional synthetic preparation. In comparison to other bioconjugation techniques, the Cadiot-Chodkiewicz reaction may be slower; however, it employs more synthetically accessible UAAs and results in a 


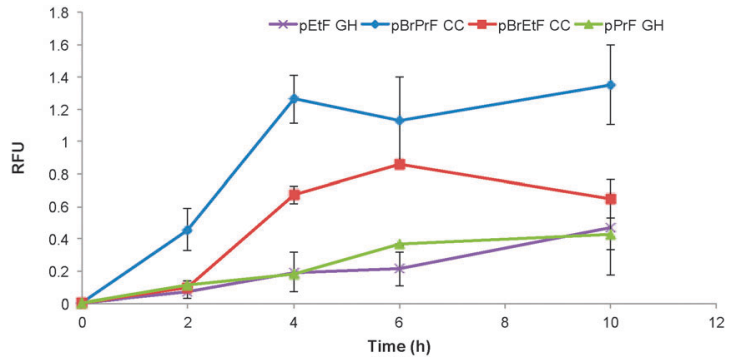

Fig. 4 Comparison of alkyne reactions. Reactions were conducted either under the described Cadiot-Chodkiewicz conditions or the Glaser-Hay conditions at $4{ }^{\circ} \mathrm{C}$ depending on incorporated UAA. Due to differences in protein levels resulting from oxidative damage, the ratio of fluorophore coupling for each data set was normalized to the 24 hour time point in order to compare coupling trends between reactions.

well-defined linear geometry primed for further reactions. Thus, the selection of the Cadiot-Chodkiewicz reaction may be dependent on the downstream application and available resources.

Overall, we have accomplished the successful application of the Cadiot-Chodkiewicz reaction to a biological context. Furthermore, we have demonstrated that the reaction can be performed with minimal protein oxidation. Finally, we have showed that the Cadiot-Chodkiewicz variant requires less harsh copper(I) concentrations, bringing the reaction near the range for in vivo use. Future work will involve optimization of conditions to increase the compatibility of the reaction with biological systems, and extension of the reaction towards in vivo applications.

\section{Notes and references}

1 G. T. Hermanson, Bioconjugate Techniques, Academic Press, London, 3rd edn, 1996.

2 K. Lang and J. Chin, Chem. Rev., 2014, 114, 4764-4806.

$3 \mathrm{H}$. Zhu and M. Snyder, Curr. Opin. Chem. Biol., 2003, 7, 55-63.

4 W. Tan, L. Sabet, Y. Li, T. Yu, P. R. Klokkevold, D. T. Wong and C. M. Ho, Biosens. Bioelectron., 2008, 24, 266-271.

5 D. Brady and J. Jordaan, Biotechnol. Lett., 2009, 31, 1639-1650.

6 F. Rusmini, Z. Zhong and J. Feijen, Biomacromolecules, 2007, 8, 1775-1789.

7 C. Liu, P. Schultz, R. Kornberg, C. Raetz, J. Rothman and J. Thorner, Annu. Rev. Biochem., 2010, 79, 413-444.

8 T. S. Young and P. G. Schultz, J. Biol. Chem., 2010, 285, 11039-11044. 9 L. Wang and P. G. Schultz, Angew. Chem., Int. Ed., 2004, 44, 34-66.
10 E. Sletten and C. Bertozzi, Angew. Chem., Int. Ed., 2009, 48, 6974-6998. 11 N. J. Agard, J. M. Baskin, J. A. Prescher, A. Lo and C. R. Bertozzi, ACS Chem. Biol., 2006, 1, 644-648.

12 Q. Wang, T. R. Chan, R. Hilgraf, V. V. Fokin, K. B. Sharpless and M. G. Finn, J. Am. Chem. Soc., 2003, 125, 3192-3193.

13 V. V. Rostovtsev, L. G. Green, V. V. Fokin and K. B. Sharpless, Angew. Chem., Int. Ed., 2002, 41, 2596-2599.

14 H. Kolb and K. Sharpless, Drug Discovery Today, 2003, 8, 1128-1137.

15 N. J. Agard, J. A. Prescher and C. R. Bertozzi, J. Am. Chem. Soc., 2004, 126, 15046-15047.

16 J. C. Jewett and C. R. Bertozzi, Chem. Soc. Rev., 2010, 39, 1272-1279.

17 J. Baskin and C. Bertozzi, QSAR Comb. Sci., 2007, 26, 1211-1219.

18 J. Kalia and R. Raines, Curr. Org. Chem., 2010, 14, 138-147.

19 J. Kalia and R. T. Raines, Angew. Chem., Int. Ed., 2008, 47, 7523-7526.

20 B. Hutchins, S. Kazane, K. Staflin, J. Forsyth, B. Felding-Habermann, V. Smider and P. Schultz, Chem. Biol., 2011, 18, 299-303.

21 S. A. Kazane, J. Y. Axup, C. H. Kim, M. Ciobanu, E. D. Wold, S. Barluenga, B. A. Hutchins, P. G. Schultz, N. Winssinger and V. V. Smider, J. Am. Chem. Soc., 2013, 135, 340-346.

22 J. S. Lampkowski, J. K. Villa, T. S. Young and D. D. Young, Angew. Chem., Int. Ed., 2015, 54, 9343-9346.

23 W. Chodkiewicz, P. Cadiot and A. Willemart, C. R. Hebd. Seances Acad. Sci., 1957, 245, 2061-2062.

24 J. Montierth, D. DeMario, M. Kurth and N. Schore, Tetrahedron, 1998, 54, 11741-11748.

25 J. Berná, S. M. Goldup, A. L. Lee, D. A. Leigh, M. D. Symes, G. Teobaldi and F. Zerbetto, Angew. Chem., Int. Ed., 2008, 47, 4392-4396.

26 M. Mowery and C. Evans, Tetrahedron Lett., 1997, 38, 11-14.

27 H. Matsuda, H. Nakanisi, T. Hosomi and M. Kato, Macromolecules, 1988, 21, 1238-1240.

28 A. Shun and R. Tykwinski, Angew. Chem., Int. Ed., 2006, 45, 1034-1057.

29 C. Glaser, Ber. Dtsch. Chem. Ges., 1896, 2, 422-424.

30 A. Hay, J. Org. Chem., 1962, 27, 3320.

31 M. Vilhelmsen, J. Jensen, C. Tortzen and M. Nielsen, Eur. J. Org. Chem., 2013, 701-711.

32 A. Hay, J. Polym. Sci., Part A: Polym. Chem., 1962, 58, 581-591.

33 J. Ueda, Y. Shimazu and T. Ozawa, Free Radical Biol. Med., 1995, 18, 929-933.

34 S. I. Lim, Y. Mizuta, A. Takasu, Y. H. Kim and I. Kwon, PLoS One, 2014, 9, e98403.

35 A. Deiters and P. G. Schultz, Bioorg. Med. Chem. Lett., 2005, 15, $1521-1524$

36 X. Nie and G. Wang, J. Org. Chem., 2006, 71, 4734-4741.

37 J. P. Marino and H. N. Nguyen, J. Org. Chem., 2002, 67, 6841-6844.

38 B. K. Raliski, C. A. Howard and D. D. Young, Bioconjugate Chem., 2014, 25, 1916-1920.

39 D. Young, T. Young, M. Jahnz, I. Ahmad, G. Spraggon and P. Schultz, Biochemistry, 2011, 50, 1894-1900.

40 D. Young, S. Jockush, N. Turro and P. Schultz, Bioorg. Med. Chem. Lett., 2011, 21, 7502-7504.

41 A. J. Link, M. K. Vink and D. A. Tirrell, J. Am. Chem. Soc., 2004, 126, 10598-10602. 